\title{
A Major External Source of Cholinergic Innervation of the Striatum and Nucleus Accumbens Originates in the Brainstem
}

\author{
Daniel Dautan, ${ }^{1,2}$ Icnelia Huerta-Ocampo, ${ }^{1}$ Ilana B. Witten, ${ }^{3}$ Karl Deisseroth,${ }^{4}$ J. Paul Bolam, ${ }^{1}$ Todor Gerdjikov, ${ }^{2}$ \\ and Juan Mena-Segovia ${ }^{1}$ \\ ${ }^{1}$ Medical Research Council Anatomical Neuropharmacology Unit, Department of Pharmacology, University of Oxford, Oxford OX1 3TH, United Kingdom, \\ ${ }^{2}$ School of Psychology, University of Leicester, Leicester LE1 9HN, United Kingdom, ${ }^{3}$ Princeton Neuroscience Institute, Princeton New Jersey 08540, and \\ ${ }^{4}$ Department of Psychiatry, Stanford University, Stanford, California 94305
}

Cholinergic transmission in the striatal complex is critical for the modulation of the activity of local microcircuits and dopamine release. Release of acetylcholine has been considered to originate exclusively from a subtype of striatal interneuron that provides widespread innervation of the striatum. Cholinergic neurons of the pedunculopontine (PPN) and laterodorsal tegmental (LDT) nuclei indirectly influence the activity of the dorsal striatum and nucleus accumbens through their innervation of dopamine and thalamic neurons, which in turn converge at the same striatal levels. Here we show that cholinergic neurons in the brainstem also provide a direct innervation of the striatal complex. By the expression of fluorescent proteins in choline acetyltransferase (ChAT)::Cre ${ }^{+}$transgenic rats, we selectively labeled cholinergic neurons in the rostral PPN, caudal PPN, and LDT. We show that cholinergic neurons topographically innervate wide areas of the striatal complex: rostral PPN preferentially innervates the dorsolateral striatum, and LDT preferentially innervates the medial striatum and nucleus accumbens core in which they principally form asymmetric synapses. Retrograde labeling combined with immunohistochemistry in wild-type rats confirmed the topography and cholinergic nature of the projection. Furthermore, transynaptic gene activation and conventional double retrograde labeling suggest that LDT neurons that innervate the nucleus accumbens also send collaterals to the thalamus and the dopaminergic midbrain, thus providing both direct and indirect projections, to the striatal complex. The differential activity of cholinergic interneurons and cholinergic neurons of the brainstem during reward-related paradigms suggest that the two systems play different but complementary roles in the processing of information in the striatum.

Key words: cholinergic; innervation; laterodorsal tegmental nucleus; pedunculopontine nucleus; striatum; topography

\section{Introduction}

The striatal complex is critically involved in movement, learning, habit formation, and reward-related behavior. Broadly differentiated into two major regions, dorsal and ventral [the latter also known as nucleus accumbens (NA)], the striatum is predominantly composed of GABAergic projection neurons and a small proportion (5-10\%) of interneurons (Graveland and DiFiglia, 1985; Kawaguchi et al., 1995; Tepper and Bolam, 2004). Among them, cholinergic interneurons provide a dense innervation of the entire striatal complex (Bolam et al., 1984; Phelps et al., 1985; Phelps and Vaughn, 1986) and have been proposed to play key

Received Nov. 29, 2013; revised Jan. 24, 2014; accepted Feb. 15, 2014.

Author contributions: D.D. and J.M.-S. designed research; D.D. and I.H.-0. performed research; I.B.W. and K.D. contributed unpublished reagents/analytic tools; D.D., I.H.-O., J.P.B., and J.M.-S. analyzed data; D.D., I.H.-O., J.P.B., T.G., and J.M.-S. wrote the paper.

This work was supported by the Medical Research Council. We thank M. Valencia for advice on data analysis and C. Etienne, E. Norman, and L. Conyers for their technical assistance.

Correspondence should be addressed to Juan Mena-Segovia, Medical Research Council Anatomical Neuropharmacology Unit, Department of Pharmacology, University of Oxford, Mansfield Road, Oxford OX1 3TH, UK. E-mail: juan.mena-segovia@pharm.ox.ac.uk.

DOI:10.1523/JNEUROSCI.5071-13.2014

Copyright $\odot 2014$ the authors $\quad 0270-6474 / 14 / 344509-10 \$ 15.00 / 0$ roles in the regulation of the activity of the local circuits (Galarraga et al., 1999; Koós and Tepper, 2002), in the presynaptic modulation of dopamine release (Rice and Cragg, 2004; Cachope et al., 2012; Threlfell et al., 2012), and in behavior (Kimura et al., 1984; Aosaki et al., 1994; Apicella et al., 1997; Blazquez et al., 2002; Morris et al., 2004; Joshua et al., 2008; Witten et al., 2010). They have been considered to be the only source of acetylcholine in the striatum (Wang et al., 2006; Ding et al., 2010; Goldberg et al., 2012).

The pedunculopontine (PPN) and the laterodorsal tegmental (LDT) nuclei located in the mesopontine tegmentum of the brainstem also contain populations of cholinergic neurons and provide widespread innervation of large regions of the brain. In the case of the PPN, its cholinergic and noncholinergic neurons innervate most basal ganglia structures (Mena-Segovia et al., 2004). The PPN and LDT have been proposed to influence activity within the striatum by two routes: (1) by innervation of midbrain dopamine neurons (Gould et al., 1989; Bolam et al., 1991; Oakman et al., 1995), in which acetylcholine modulates the activity of dopamine neurons (Lacey et al., 1990; Zhang et al., 2005) and leads to increased dopamine release in the striatal complex (Hernández-López et al., 1992; Blaha and Winn, 1993; Blaha et 
al., 1996); (2) cholinergic neurons of the brainstem innervate neurons of the intralaminar and midline thalamus (Smith et al., 1988; Kobayashi and Nakamura, 2003; Mena-Segovia et al., 2008; Parent and Descarries, 2008) that in turn project to the striatal complex (Erro et al., 1999).

An additional route by which brainstem cholinergic neurons could influence the striatum is by direct innervation. Indeed, data from earlier tract-tracing studies suggest that this may be the case (Saper and Loewy, 1982; Smith and Parent, 1986; Hallanger and Wainer, 1988; Nakano et al., 1990). In view of the importance of cholinergic mechanisms in the regulation of dopamine release (Cachope et al., 2012; Threlfell et al., 2012) and network activity in the striatum (Carrillo-Reid et al., 2009; Goldberg et al., 2012), we aimed to determine whether cholinergic neurons in the brainstem directly innervate the striatum. We used a Cre-driver line to genetically target cholinergic neurons and induce the expression of a fluorescent marker in their axons (Witten et al., 2011). Our combined approach reveals that the dorsal striatum and NA receive a dense and highly organized cholinergic innervation arising in the brainstem.

\section{Materials and Methods}

Animals. Adult (250-450 g) Long-Evans (LE) wild-type and ChAT::Cre ${ }^{+}$ (Witten et al., 2011) male $(n=29)$ and female $(n=5)$ rats were used for all experiments. Rats were maintained on a $12 \mathrm{~h}$ light/dark cycle (light on 7:00 A.M.) and ad libitum access to water and food. All procedures were performed in accordance with the Society for Neuroscience policy on the use of animals in neuroscience and the Animals (Scientific Procedures) Act, 1986 (United Kingdom), under the authority of a Project License approved by the Home Office and the local ethical committee of the University of Oxford.

Stereotaxic injections. All surgical procedures were performed during deep isoflurane anesthesia ( $2 \%$ in $\mathrm{O}_{2}$; Isoflo; Schering-Plough). For the anterograde tracing studies, LE ChAT::Cre ${ }^{+}$rats $(n=9)$ were injected with adeno-associated virus serotype 2 (AAV2) carrying the fusion genes for channelrhodopsin 2 (ChR2) and yellow fluorescent protein (YFP) (AAV2-EF1a-DIO-hChR2-YFP; Gene Therapy Center Virus Vector Core, University of North Carolina). The AAV2-EF1a-DIO-hChR2YFP was injected in the rostral part of the PPN [ $400 \mathrm{nl}$ over $10 \mathrm{~min}$; from bregma in $\mathrm{mm}$ : anteroposterior (AP), -6.8 ; mediolateral $(\mathrm{ML}),+1.8$; dorsoventral (DV), -7.5 ventral of the dura; $n=3$ ], the caudal part of the PPN (400 $\mathrm{nl}$ over $10 \mathrm{~min}$; from bregma in $\mathrm{mm}$ : AP, -7.8 ; ML, +1.8; DV, -6.5 ventral of the dura; $n=3$ ), or the LDT ( $300 \mathrm{nl}$ over $10 \mathrm{~min}$; from bregma in $\mathrm{mm}$ : $\mathrm{AP},-8.5 ; \mathrm{ML},+0.9 ; \mathrm{DV},-6.0$ ventral of the dura; $n=3)$. An additional two rats were injected into the dorsolateral striatum (from bregma in mm: AP, +0.5 ; ML, +2.4 ; DV, -4.5 ventral of the dura) to label cholinergic interneurons within the striatum.

For the retrograde tracing studies, wild-type LE rats $(n=10)$ were injected with cholera toxin b (CTb; $2.5 \%$ in distilled water, $400 \mathrm{nl}$ over 10 min; Sigma-Aldrich) and Fluorogold (FG; $2.0 \%$ in distilled water, $300 \mathrm{nl}$ over $10 \mathrm{~min}$; Fluorochrome), each tracer in one of the following areas: the lateral striatum (as above), the medial striatum (from bregma in $\mathrm{mm}$ : $\mathrm{AP},+1.0 ; \mathrm{ML},+1.4 ; \mathrm{DV},-4.5$ ventral of the dura), the lateral shell of the $\mathrm{NA}$ (from bregma in $\mathrm{mm}$ : AP, $+1.2 ; \mathrm{ML},+2.7 ; \mathrm{DV},-6.8$ ventral of the dura), the medial shell of the NA (from bregma in mm: AP, +1.5; ML, +0.9 ; DV,-7.2 ventral of the dura), or the medial core of the NA (from bregma in $\mathrm{mm}$ : AP, $+1.5 ; \mathrm{ML},+0.9 ; \mathrm{DV},-6.5$ ventral of the dura). Each rat received two injections, and the combinations of tracers/targets were varied. Finally, for the combined retrograde/anterograde tracing studies, wild-type LE rats $(n=6)$ were injected with AAV-EF1a-DIO-WGACre-mCherry in the dorsolateral striatum or the NA and with AAV2EF1a-DIO-hChR2-YFP in the rostral PPN or LDT, respectively. The wheat germ agglutinin (WGA)-Cre fusion protein was expressed by the AAV-EF1a-DIO-WGA-Cre-mCherry, transcellularly transported to the afferent neurons, and retrogradely transported to the soma, in which it produced the recombination of AAV2-EF1a-DIO-hChR2-YFP and the expression of YFP, thus specifically labeling the brainstem neurons projecting to the striatum and their axon collaterals (Gradinaru et al., 2010). All injections were made using designated $1 \mu \mathrm{l}$ syringes (SGE Analytical Science) at rate of $40 \mathrm{nl} / \mathrm{min}$ and a postinjection diffusion time of $5 \mathrm{~min}$. Approximately 4 weeks later, after the AAV2-EF1a-DIOhChR2-YFP virus injection, $10-15 \mathrm{~d}$ after the tracers injections, and 2-4 weeks after the AAV-EF1a-DIO-WGA-Cre-mCherry plus AAV2EF1a-DIO-hChR2-YFP injections, the rats were given a lethal dose of pentobarbital $(200 \mathrm{mg} / \mathrm{kg}$, i.p.) and perfused transcardially with $0.05 \mathrm{M}$ PBS, pH 7.4, followed by $300 \mathrm{ml}$ of $4 \% \mathrm{w} / \mathrm{v}$ paraformaldehyde in phosphate buffer $(0.1 \mathrm{~m}, \mathrm{pH} 7.4)$ containing $0.1 \%$ glutaraldehyde (TAAB Laboratories). Brains were stored in PBS at $4^{\circ} \mathrm{C}$ until sectioning. To corroborate the findings from this part of the study, an additional group of wild-type LE rats $(n=4)$ were used in a double retrograde labeling experiment. FG was injected into the NA $(500 \mathrm{nl}$ over $10 \mathrm{~min}$; from bregma in $\mathrm{mm}$ : AP, +1.5; ML, +0.2; DV, -7.0 ventral of the dura), and $\mathrm{CTb}$ was injected in either the ventral tegmental area (VTA; $300 \mathrm{nl}$ over $10 \mathrm{~min}$; from bregma in $\mathrm{mm}$ : AP, $-5.2 ; \mathrm{ML},+0.9$; DV, -7.5 ventral of the dura) or the mediodorsal thalamus ( $500 \mathrm{nl}$ over $10 \mathrm{~min}$; from bregma in $\mathrm{mm}$ : AP, -3 ; ML, +1.4 ; DV, -5.0 ventral of the dura). Retrogradely labeled neurons were revealed after perfusion fixation as described above.

Immunohistochemistry. Sagittal sections of the right hemispheres were cut at $50 \mu \mathrm{m}$ thickness in PBS using a vibratome (VT1000S; Leica). For each brain, the sites of injection were verified (see below), and only those with on-target injections were processed further. For the anterograde tracing study, sections containing the entire striatum and NA (4.6-1.13 $\mathrm{mm}$, from the midline) were incubated overnight in a solution containing antibodies against green fluorescent protein (GFP; also detects YFP; 1:1000, raised in rabbit, A21311; Invitrogen) and either ChAT (raised in goat, 1:500 dilution; Millipore) or tyrosine hydroxylase (TH; to define the accumbens and striatal borders; raised in chicken, 1:500 dilution; Abcam) in 1\% normal donkey serum (NDS) and $0.03 \%$ Triton X-100 in PBS. After several washes in PBS, the sections were incubated for a minimum of $4 \mathrm{~h}$ in an Alexa Fluor 488-conjugated donkey anti-rabbit antibody (1:1000; Life Technologies) and either Cy3-conjugated donkey anti-chicken (1:1000; Jackson ImmunoResearch) or Cy3-conjugated donkey anti-goat antibodies (1:1000; Jackson ImmunoResearch). Additional sections were incubated with an antibody against the vesicular acetylcholine transporter (VAChT; raised in guinea pig, 1:500 dilution in $1 \%$ NDS and $0.03 \%$ Triton X-100 in PBS, AB1588; Millipore Bioscience Research Reagents) to detect the presence of VAChT in the YFP-labeled axons. Some sections were incubated with antibodies against $\mu$-opioid receptor (MOR; raised in guinea pig, $\mathrm{AB} 1774$; Millipore) to define the striosomes (Graybiel and Ragsdale, 1978). These incubations were followed by their corresponding fluorophore-conjugated secondary antibodies, as described above.

Sections of the brains that had received tracers injections were first incubated in antibodies against ChAT (1:500) and CTb (raised in mouse, 1:500 dilution in 1\% NDS and 0.3\% Triton X-100 in PBS; Abcam), washed in PBS, and incubated in Cy3-conjugated donkey anti-mouse antibody (1:1000; Jackson ImmunoResearch) and Cy5-conjugated donkey anti-goat antibody (1:1000; Jackson ImmunoResearch). For FG detection, no additional processing was required.

The sections were mounted on slides using Vectashield and then examined in a fluorescent (ImagerM2; Zeiss) or confocal (LSM-510; Zeiss) microscope using the following filters: $504 \mathrm{~nm}$ for FG and Alexa Fluor488, $560 \mathrm{~nm}$ for Cy3, and $650 \mathrm{~nm}$ for Cy5 (20×, 0.8 numerical aperture dry objective or $40 \times, 1.4$ numerical aperture oil immersion). Confocal images were processed using Huygens Professional deconvolution software (version 4.1; Scientific Volume Imaging) with a maximum of 40 iterations. The brightness and contrast of captured images were adjusted in Photoshop (Adobe Systems). The distributions of labeled axons in the dorsal striatum and NA and retrogradely labeled neurons in the PPN and LDT were digitized offline using StereoInvestigator (MicroBrightField).

Analysis of the distribution of cholinergic axons. In sections from ChAT::Cre ${ }^{+}$rats, each of the injection groups (rostral PPN, caudal PPN, or LDT) were analyzed separately. The level of expression of YFP-labeled axons was initially evaluated in the striatum and NA across all levels. To provide a quantitative estimation of the distribution of cholinergic pro- 
jections across the different groups, three sections per animal were selected corresponding to the following ML levels (from the midline, in $\mathrm{mm}$ ): 3.18, 2.10, and 1.13 (Paxinos and Watson, 1986). The striatum, NA, and olfactory tubercle were outlined and subdivided into $40 \mu \mathrm{m}^{2}$ bins using StereoInvestigator. Each bin containing at least one YFPpositive labeled axon was marked as positive. The markers were interpolated into an $80 \mu \mathrm{m}^{2}$-bin grid that was averaged across the same ML levels of animals from the same group using MATLAB. Data were then normalized to obtain the probability of innervation. This was plotted as a color map to represent the regions of higher density of cholinergic projections.

Analysis of the distribution of retrogradely labeled neurons. The number of neurons in the rostral PPN, caudal PPN, and LDT projecting to the lateral and medial regions of the dorsal striatum and lateral, medial shell, and core of the NA were quantified according to their rostrocaudal distribution. Neurons within the PPN and LDT, as delimited by the ChATimmunopositive cell bodies, were classified as follows: (1) $\mathrm{ChAT}^{+} /$ $\mathrm{CTb}^{+}$; (2) $\mathrm{ChAT}^{+} / \mathrm{FG}^{+}$; (3) $\mathrm{ChAT}^{-} / \mathrm{CTb}^{+}$; or (4) $\mathrm{ChAT}^{-} / \mathrm{FG}^{+}$. Three representative $\mathrm{ML}$ levels of the brainstem were selected for the analysis corresponding to $1.55,1.13$, and $0.9 \mathrm{~mm}$ from the midline (Paxinos and Watson, 1986). To define the boundaries between rostral PPN and caudal PPN, we adapted a method based on the subdivision of the PPN into equally spaced segments in the sagittal plane using concentric circles, as described previously (Mena-Segovia et al., 2009). Thus, using the center of the substantia nigra pars reticulata $(\mathrm{SNr})$ as a reference point, two equally sized segments of $1400 \mu \mathrm{m}$ width were defined. The first segment represents the rostral PPN (0.6-2.0 $\mathrm{mm}$ from the midpoint of the SNr), and the second segment represents the caudal PPN $(2.0-3.3$ $\mathrm{mm}$ from the midpoint of the $\mathrm{SNr}$ ). The LDT neurons were quantified in a single segment ( $>3.3 \mathrm{~mm}$ from the $\mathrm{SNr}$ ). Results are expressed as total numbers of neurons that are positive for each of the tracers and the normalized number of retrogradely traced neurons that are immunopositive for ChAT.

Preparation of tissue and immunohistochemistry for electron microscopy. A separate series of sections from ChAT::Cre ${ }^{+}$rats were incubated in a cryoprotectant solution ( $0.05 \mathrm{~m}$ phosphate buffer, $25 \%$ sucrose, and $10 \%$ glycerol) overnight and then freeze-thawed three times in liquid nitrogen to increase penetration of the reagents. The sections were washed thoroughly and then incubated in 10\% normal goat serum (NGS; Vector Laboratories) in PBS (NGS-PBS) for $2 \mathrm{~h}$ at room temperature. All sections were immunolabeled to reveal YFP-containing structures using a biotinylated antibody against GFP, raised in goat (BA-0702; Vector Laboratories), at a dilution of 1:500 in 1\% NGS-PBS overnight at room temperature. This was followed by incubation in an avidin-biotin-peroxidase complex (ABC Elite; Vector Laboratories) for 3-4 h at room temperature. The sections were then washed in PBS, followed by washes in Tris buffer (TB; $0.5 \mathrm{M}, \mathrm{pH} 8)$ and then transferred to diaminobenzidine solution ( 0.025 and $0.5 \%$ nickel ammonium sulfate in $\mathrm{TB}$ ) for $15 \mathrm{~min}$. The peroxidase reaction was initiated by the addition of $\mathrm{H}_{2} \mathrm{O}_{2}$ to a final concentration of $0.01 \%$. The reaction was allowed to continue for $\sim 10$ min and was stopped by several washes in TB and then PB. The sections were postfixed in 1\% osmium tetroxide in $\mathrm{PB}$ for $25 \mathrm{~min}$. They were washed in $\mathrm{PB}$ and dehydrated through a graded series of dilutions of ethanol with $1 \%$ uranyl acetate (TAAB Laboratories) included in the $70 \%$ ethanol solution to increase contrast in the electron microscope. After absolute ethanol, sections were washed twice in propylene oxide (Sigma) for $15 \mathrm{~min}$ and placed in resin overnight at room temperature (Durcupan ACM; Fluka). They were then mounted in resin on glass microscope slides and polymerized at $60^{\circ} \mathrm{C}$ for $48 \mathrm{~h}$. All sections were examined in the light microscope to determine the extent and localization of the labeling. The selected regions were cut from the slides and reembedded in a cylinder of resin for subsequent ultrathin sectioning for the electron microscope.

Electron microscopic analysis. Serial sections $(\sim 55 \mathrm{~nm})$ were cut on an ultramicrotome (Leica EM UC6; Leica Microsystems) and collected on pioloform-coated, single-slot copper grids (Agar Scientific). The sections were lead stained for 5-7 min and examined in a Philips CM100 EM. YFP-immunoreactive structures were identified by the electron-dense peroxidase reaction product that adhered to the internal surface of the plasmalemma and the outer membrane of organelles. Axonal profiles were identified by the presence of synaptic vesicles and often mitochondria. YFP-positive axonal profiles were examined in serial sections and synapses identified. For each synapse, the postsynaptic target was characterized. Synapses of the asymmetrical type (Gray's type 1) were characterized as such by the presence of presynaptic vesicle accumulation, a thick postsynaptic density, a widened synaptic cleft, and cleft material, whereas symmetric synapses (Gray's type 2) possessed a much less pronounced postsynaptic density. Structures that did not fulfill these criteria were not considered as synaptic junctions. The digital images were analyzed using NIH Image J, and they were adjusted for contrast and brightness using Adobe Photoshop CS5.1 (Adobe Systems).

\section{Results}

\section{Innervation of the striatum and NA by cholinergic axons originating in the PPN and LDT nuclei}

Three to 4 weeks after infection with the AAV vector in the ChAT::Cre ${ }^{+}$rats, clusters of cholinergic neurons, identified by immunofluorescent labeling for the YFP, were found around the sites of injection. Cell bodies, dendrites, and local axonal arbors were labeled, and the clusters of labeled neurons ranged from 0.5 to $1 \mathrm{~mm}$ in diameter in the sagittal plane. The axons of the brainstem cholinergic neurons gave rise to widespread projections in the form of beaded axons in the midbrain and forebrain, including the superior colliculus, substantia nigra, thalamus, subthalamic nucleus and globus pallidus (external), and ventral pallidum. In addition to this, each of the injections in the PPN or LDT gave rise to YFP-positive axons that occupied most regions of the striatum, NA, and substriatal structures, and their distribution correlated with the region of the brainstem injected (Fig. 1; see below). The labeled axons gave rise to frequent varicosities, could follow long, straight paths or give rise to tortuous arborizations (Figs. 2-4) that mostly avoided patches/striosomes (see below). Furthermore, immunofluorescence labeling for VAChT of some of the YFP-positive axons and boutons confirmed the cholinergic nature of the projection (Figs. $2 C, 3 C, 4 C$ ).

The cholinergic projection from the PPN and LDT to the striatal complex was topographically organized such that the rostral part of the cholinergic brainstem innervated more abundantly the lateral levels of the striatum, and the caudal part innervated more abundantly the medial and ventral levels of the striatum. The AAV injections in the rostral PPN produced areas of dense innervation (patches) mainly in the dorsolateral striatum (Figs. 1, 2A, 2E). In contrast, deposits in the caudal PPN gave rise to smaller areas of innervation that were sparser and distributed over different levels of the dorsal striatum and NA (Figs. 1, $3 A, E)$. Cholinergic axons from the LDT showed a level of innervation comparable with that of the rostral PPN, but it was concentrated in the dorsomedial striatum and NA (Figs. 1, 4A,E). In each case, regions of axonal arborizations in the striatal complex avoided the patches/striosome as defined by the high level of expression of MOR immunoreactivity (Figs. 2B, 3B, 4B). Furthermore, in each case, electron microscopic (EM) analysis revealed that the axonal varicosities formed synaptic connections (Figs. 2D, 3D, 4D). These data reveal a topographically organized cholinergic projection originating in the brainstem that innervates wide areas of the dorsal striatum and NA, forming synaptic contacts with different postsynaptic structures.

\section{EM observations}

EM analysis of the striatum revealed that the axons of brainstem cholinergic neurons gave rise to vesicle-filled boutons that often contained one or more mitochondria and formed synaptic contacts with spines (Figs. 3D, 4D) and dendritic shafts (Fig. 2D). 


\section{PPNr PPNC LDT}
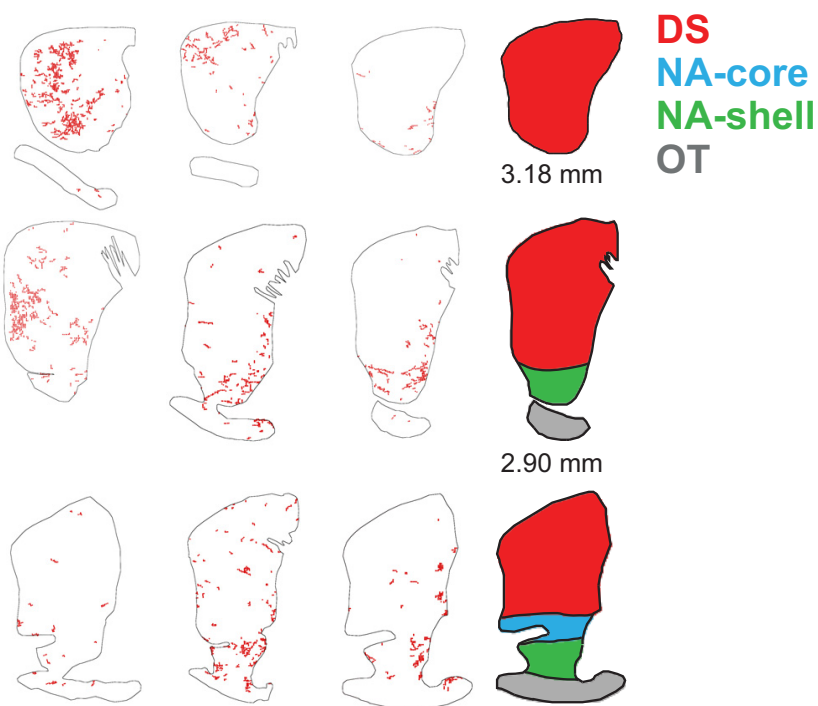

$2.10 \mathrm{~mm}$
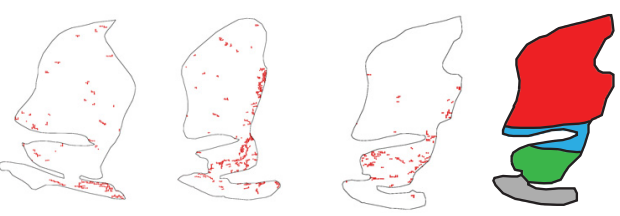

$1.90 \mathrm{~mm}$
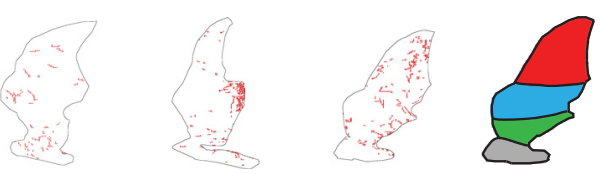

$1.40 \mathrm{~mm}$
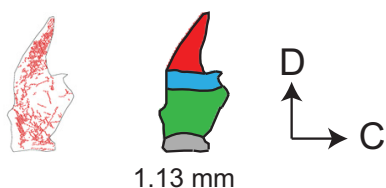

Figure 1. Distribution of cholinergic axons in the striatal complex after viral vector injections into different groups of cholinergic neurons in the brainstem. Plots in the sagittal plane of the distribution of cholinergic axonal profiles from three representative animals that received viral vector injections in the rostral PPN (PPNr), the caudal PPN (PPNc), and the LDT. Injections in the rostral PPN gave rise to a dense innervation of the lateral striatum. Injections in the caudal PPN gave rise to a sparser innervation, with a tendency to innervate more medial regions of the striatum and parts of the NA. In contrast, injections into the LDT led to dense labeling in the most medial striatum and the NA core. Each injection led to labeling in the olfactory tubercle. Each red dot represents at least one immunopositive axonal profile in a bin of $40 \mu \mathrm{m}^{2}$. The corresponding template (right) illustrates the ML levels (millimeters from the midline) and subdivisions of the striatum (DS, dorsal striatum), NA core (NA-core) and shell (NA-shell), and olfactory tubercle (OT) according to the stereotaxic rat brain atlas of Paxinos and Watson (1986). D, Dorsal; C, caudal. Scale bar, $2 \mathrm{~mm}$.

Synapses were mostly asymmetric (Gray's type 1; Figs. $2 D, 4 D$, $5 A, B, E, F)$; in some cases, the postsynaptic density was very prominent (similar to cortical and thalamic synapses) but on other occasions was less dense and more diffuse (Fig. 5B). Symmetrical synapses were less frequently observed (Gray's type 2; Figs. $3 D, 5 C$ ). Serial section analysis revealed that synaptic incidence varied depending on the origin (highest for LDT axons and lowest for caudal PPN axons).

The deposits of the viral vector in the striatum led to the labeling of striatal cholinergic neurons and their local axon col-
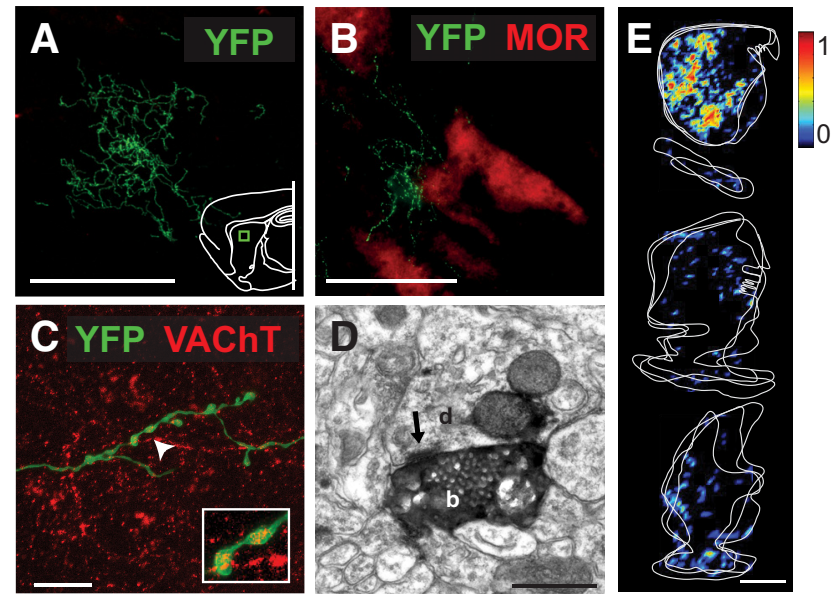

Figure 2. Cholinergic axons arising in the rostral PPN preferentially innervate the lateral striatum. $\boldsymbol{A}$, YFP-immunopositive axons arising from cholinergic neurons after the deposit of AAV2-EF1a-DI0-hChR2-YFP in the rostral PPN of a ChAT::Cre ${ }^{+}$rat. The labeled cholinergic axons formed dense regions of innervation in the dorsolateral striatum. The green box in the outline represents the area in which the image was acquired. $B$, MOR immunolabeling to identify the striosomes. The distribution of the cholinergic axons was primarily confined to the MOR-negative matrix compartment. $C$, The cholinergic axons gave rise to a large number of varicosities, some of which were identified as VAChT immunopositive (arrowhead; see inset). $\boldsymbol{D}$, Electron micrograph of a YFP-immunopositive cholinergic bouton (b) forming an asymmetric (Gray's type 1) synapse (arrow) with a dendritic shaft (d).E, Probability plot, calculated from all animals $(n=3)$ at three different ML levels $(3.18,2.10$, and $1.13 \mathrm{~mm}$ from the midline, from top to bottom), of cholinergic axons arising in the rostral PPN. Cholinergic axons from this region of the PPN were more densely distributed in the lateral aspects of the dorsal striatum. Scale bars: $A, 250 \mu \mathrm{m} ; \boldsymbol{B}, 250 \mu \mathrm{m} ; \boldsymbol{C}, 10 \mu \mathrm{m} ; \boldsymbol{D}, 0.4 \mu \mathrm{m} ; \boldsymbol{E}, 1000 \mu \mathrm{m}$.
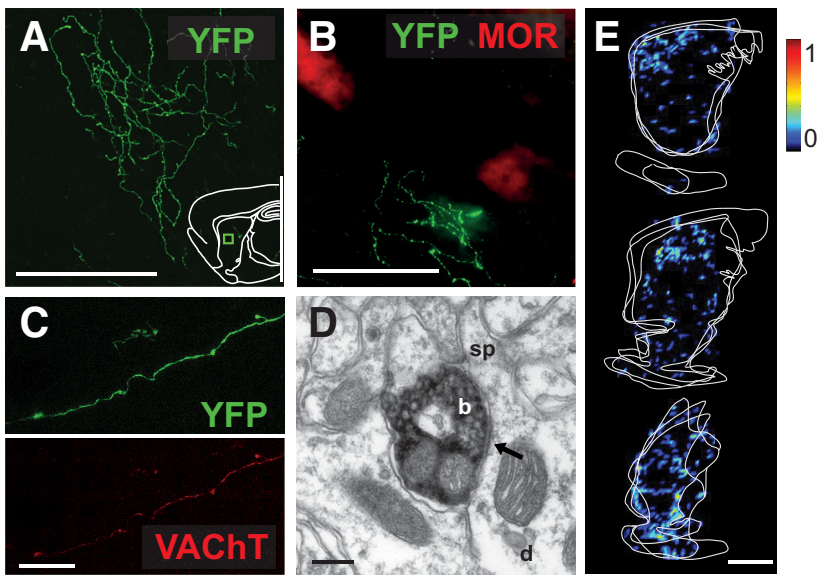

Figure 3. Cholinergic axons arising in the caudal PPN project diffusely across the striatum and NA. A, YFP-immunopositive axons arising from cholinergic neurons in the caudal PPN form small patches mainly in the dorsal part of the striatum. The green box in the outline represents the area in which the image was acquired. $\boldsymbol{B}$, Cholinergic axons from caudal PPN also avoided the striosomes. $\boldsymbol{C}$, These cholinergic axons (top) gave rise to a smaller number of varicosities than those of the rostral PPN but were always immunopositive for VAChT (bottom). D, Electron micrograph of an immunopositive cholinergic (YFP-positive) bouton (b) establishing symmetric (Gray's type 2) synaptic contact (arrow) with the neck or base of a dendritic (d) spine (sp). $\boldsymbol{E}$, Probability plot showing that cholinergic axons arising in the caudal PPN have a lower density distribution than those arising in the rostral PPN, with higher values in the lateral striatum, mainly in the more dorsal regions, and in the NA core and shell $(n=3$; at three different ML levels as in Fig. 2). Scale bars: $\boldsymbol{A}, 250 \mu \mathrm{m} ; \boldsymbol{B}, 250 \mu \mathrm{m} ; \boldsymbol{C}, 10 \mu \mathrm{m} ; \boldsymbol{D}, 0.2 \mu \mathrm{m} ; \boldsymbol{E}, 1000 \mu \mathrm{m}$.

laterals (Fig. 5D). EM analysis of 39 cholinergic synapses derived from interneurons revealed that approximately one-third form asymmetric synapses (Fig. 5E, F; including those with prominent and those with less prominent postsynaptic densities), and two- 

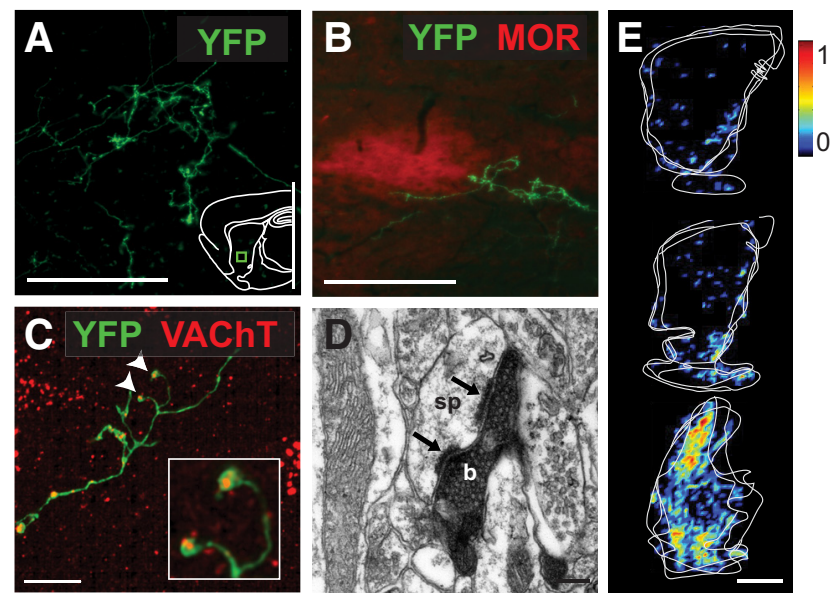

Figure 4. Cholinergic axons arising in the LDT preferentially innervate the medial striatum and the NA core. $A$, YFP-immunopositive axons from cholinergic neurons in the LDT form dense regions of innervation in the most medial levels of the striatum and NA core (depicted here). The green box in the outline represents the area in which the image was acquired. $\boldsymbol{B}$, MOR immunolabeling in the NA shell revealed that cholinergic axons from the LDT tend to avoid striosomes. C, Cholinergic axons from the LDT formed large en passant varicosities that were immunopositive for VAChT (arrowheads). D, Electron micrograph of a cholinergic axon bouton (YFP-immunopositive; b) forming asymmetric (Gray's type 1) synapses (arrows) with a spine (sp). $\boldsymbol{E}$, Probability plot showing that cholinergic axons arising in the LDT were more densely distributed in the medial striatum and the core and medial shell of the NA ( $n=3$; at three different ML levels as in Figs. 2, 3). Scale bars: $\boldsymbol{A}, 250 \mu \mathrm{m} ; \boldsymbol{B}, 100 \mu \mathrm{m} ; \boldsymbol{C}, 10 \mu \mathrm{m} ; \boldsymbol{D}, 0.2 \mu \mathrm{m} ; \boldsymbol{E}$, $1000 \mu \mathrm{m}$.

thirds formed symmetrical synapses (Fig. $5 G$ ). This contrasts to cholinergic synapses derived from the rostral PPN, of which $82 \%$ formed asymmetric synapses and $18 \%$ formed symmetrical synapses $(n=22$; Fig. $5 H)$. Similar differences in the postsynaptic targets of rostral PPN-derived and striatal interneuron-derived cholinergic synapses were observed. Thus, $\sim 74 \%$ of those derived from the rostral PPN contacted dendritic shafts and $\sim 26 \%$ contacted spines, whereas the figures were $\sim 46 \%$ in contact with shafts, $\sim 49 \%$ with spines, and $\sim 5 \%$ with perikarya for synapses derived from cholinergic interneurons. Overall, these findings suggest that cholinergic axons derived from neurons in the brainstem, like striatal cholinergic interneurons, make synaptic contact with the spines of medium spiny neurons, and the asymmetric synapses on dendritic shafts suggest that they also contact striatal interneurons.

\section{Neurons retrogradely labeled from the dorsal striatum and NA are topographically organized in the brainstem}

To extend the data from the anterograde tracing experiments using viral vectors in transgenic rats, we injected two retrograde tracers in wild-type animals. We injected a combination of targets that included the dorsolateral and dorsomedial areas of the striatum and the core, medial shell, and lateral shell of the NA. The presence and distribution of retrogradely labeled neurons were analyzed in the PPN and LDT. Immunofluorescence for ChAT was used to define the borders of the brainstem structures and to quantify the proportion of neurons projecting to the striatal complex that were cholinergic. The results confirm the presence of a topographical projection from the cholinergic neurons of the brainstem to the forebrain (Fig. 6). Injections in the dorsolateral striatum $(n=3)$ resulted in retrogradely labeled neurons mainly in the rostral and caudal PPN (Fig. $6 A, C$ ) and $>70 \%$ of the retrogradely labeled neurons were ChAT immunopositive (Table 1), whereas injections in the dorsomedial striatum $(n=$
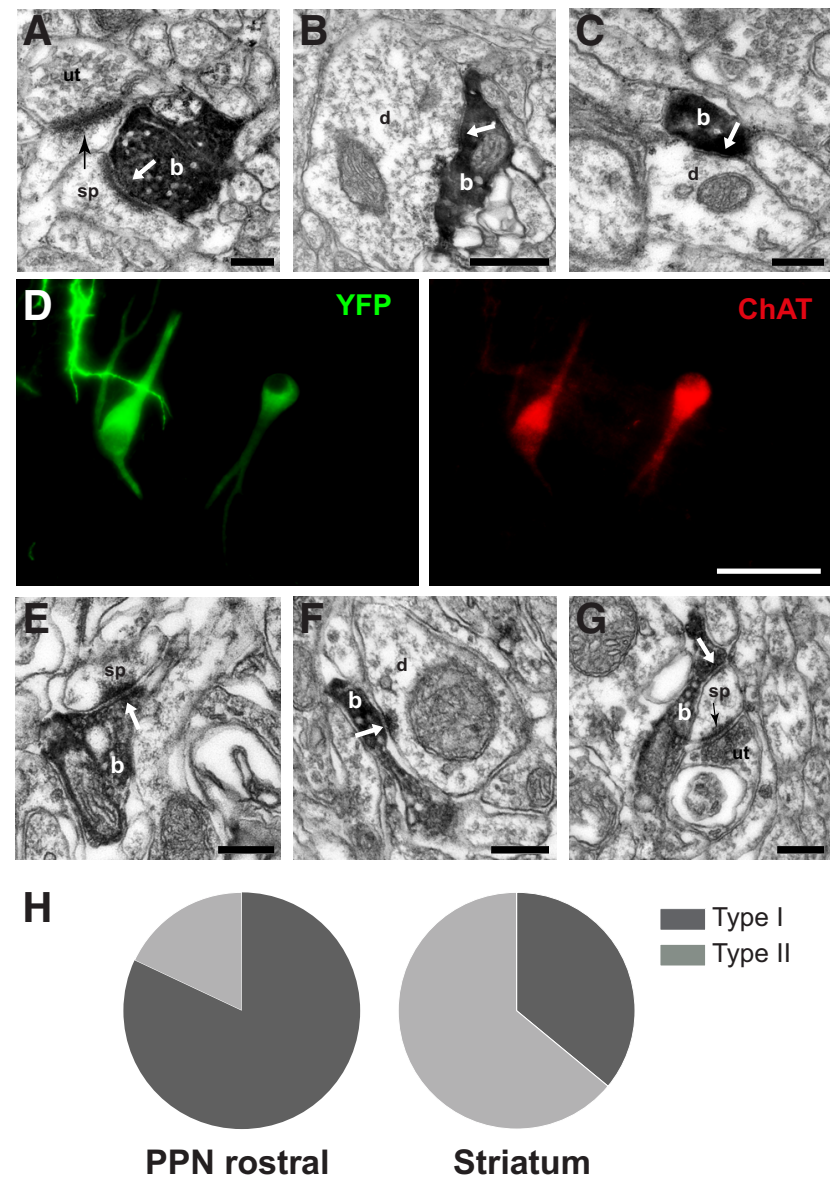

Figure 5. Morphology of synapses differentiates brainstem cholinergic from cholinergic interneurons contacts. $\boldsymbol{A}-\boldsymbol{C}$, Electron micrographs of cholinergic boutons (b) arising from the rostral PPN (YFP-immunopositive) forming asymmetric (Gray's type 1) synapses with prominent $(\boldsymbol{A})$ and moderate $(\boldsymbol{B})$ postsynaptic densities onto a spine (sp) and dendritic shaft (d), respectively. C, A PPN cholinergic terminal forming a symmetrical (Gray's type 2) synapse with a dendritic shaft. D, YFP-immunopositive cell bodies from striatal cholinergic interneurons and their axons after the deposit of AAV2-EF1a-DIO- hChR2-YFP in the dorsolateral striatum of a ChAT::Cre ${ }^{+}$rat. ChAT immunolabeling confirms their cholinergic nature. $\boldsymbol{E}, \boldsymbol{F}$, Electron micrographs of cholinergic boutons arising from striatal cholinergic interneurons (YFPimmunopositive) establishing asymmetrical synapses that possess prominent $(\boldsymbol{E})$ and less prominent $(\boldsymbol{F})$ postsynaptic densities onto a spine and dendritic shaft, respectively. $\boldsymbol{G}$, A striatal cholinergic bouton forming a symmetrical (Gray's type 2) synapse with a spine. Note the unlabeled terminals (ut) forming synapses (black arrows) with unlabeled spines in $\boldsymbol{A}$ and $\mathbf{G}$. $\boldsymbol{H}$, Synapses from the rostral PPN are predominantly asymmetric, whereas the synapses from the cholinergic interneurons are predominantly symmetric. Scale bars: $A, C, E-G, 0.2 \mu \mathrm{m} ; \boldsymbol{B}, 0.5$ $\mu \mathrm{m} ; \boldsymbol{D}, 50 \mu \mathrm{m}$.

3) led to labeling in the caudal PPN and LDT (Fig. 6C), with very little labeling in the rostral PPN (Fig. 6A). In this case, $62-76 \%$ of the labeled neurons were ChAT immunopositive (Table 1). Injections in the core and medial shell of the NA ( $n=4$ and $n=3$, respectively) led to dense labeling particularly concentrated in the LDT (Fig. $6 B, C$ ), with some labeling, albeit much sparser, in the caudal PPN. In contrast, injections in the lateral shell $(n=4)$ produced widespread labeling across the PPN and, to a minor degree, the LDT (Fig. 6C). The proportion of neurons retrogradely labeled from the NA that were ChAT immunopositive was more variable (41-78\%) than those labeled from the dorsal striatum. The fact that a proportion of the projection neurons were not ChAT positive suggests that noncholinergic neurons (i.e., glutamatergic and 
GABAergic) are also involved in the striatal projection pathways (Table 1).

\section{Axon collaterals of striatal-projecting neurons in the brainstem also innervate the dopaminergic midbrain and the thalamus}

Cholinergic neurons of the brainstem are known to innervate dopaminergic nigrostriatal/mesolimbic regions and the intralaminar thalamic nuclei (for review, see Martinez-Gonzalez et al., 2011), and as such have the potential to modulate two of the most significant inputs to the striatal complex. To determine whether it is the same brainstem neurons that innervate the striatal complex that, via collaterals, also innervate midbrain and thalamic targets, we injected a viral vector associated with the WGA-Cre fusion protein (AAV-EF1a-DIO-WGA-Cre-mCherry) in the NA core of wild-type rats $(n=3)$. This produced transcellular trafficking of the WGA-Cre fusion protein that was retrogradely transported to the cell body (Gradinaru et al., 2010; Xu and Südhof, 2013). In combination with the injection of a Cre-dependent vector in the LDT, it induced the expression of the YFP only in those brainstem neurons projecting to the NA that were retrogradely labeled with the WGA-Cre. YFP expressed by these neurons was distributed throughout their somato-dendritic and axonal arbors including their axon collaterals (Fig. 7A). These targets (NA core and LDT) were selected on the basis of our previous retrograde and anterograde results (Figs. 4, $6 B)$. We detected the presence of YFP-positive neurons in the LDT (Fig. 7B), of which the majority were immunopositive for ChAT, consistent with the other experimental approaches. In addition, we detected the presence of YFP-positive axons in the NA core, spread over the region in which the vector carrying the WGA-Cre sequence was injected. Neurons of the LDT gave rise to abundant collaterals that were detected in the VTA (Fig. 7C,D) and the midline thalamus (Fig. 7E), suggesting that LDT neurons that innervate the NA core also innervate the VTA and thalamus. This combined approach was not possible in the rostral PPN because of the proximity of dopamine neurons in the substantia nigra pars compacta $(\mathrm{SNc})$, which project densely to the dorsal striatum. Animals in which one of the two targets was missed did not produce any detectable labeling of cell bodies or axons. The time between injections and perfusion was shortened compared with previous studies (see Materials and Methods; Gradinaru et al., 2010; Xu and Südhof, 2013) to minimize the possibility of transynaptic retrograde labeling of second-order neurons at the level of the thalamus and the VTA. However, because we cannot rule out this possibility entirely, we performed additional experiments using double retrograde tracer injections into the NA core and either the VTA $(n=2)$ or the mediodorsal thalamus $(n=2)$. We observed a large number of neurons retrogradely labeled from all three structures, and the majority of them were immunopositive for ChAT (68\% for NA, $60 \%$ for VTA, and $77 \%$ for
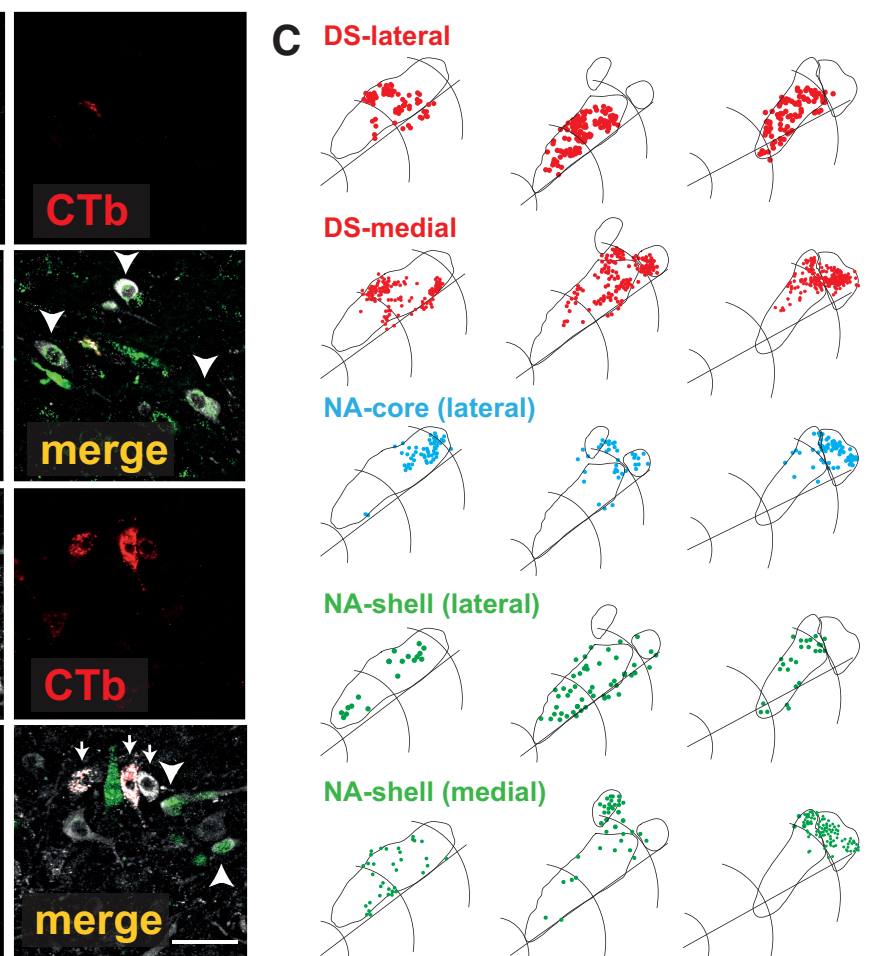

NA-core (lateral)
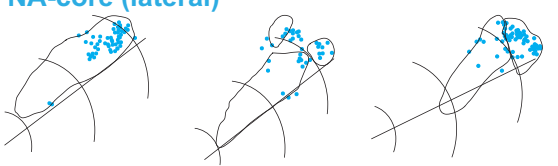

NA-shell (lateral)
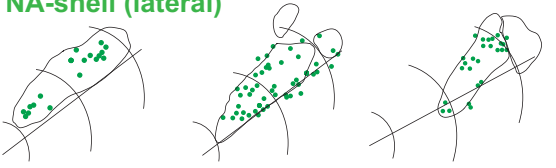

NA-shell (medial)
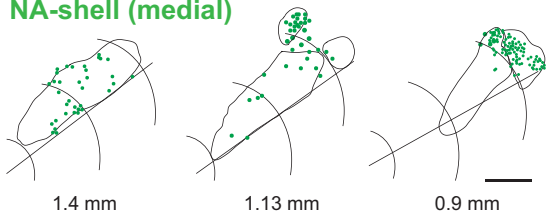

Figure 6. Retrograde labeling from the striatum and NA shows a topographical distribution of cholinergic neurons in the , $\boldsymbol{A}, \boldsymbol{B}$, Confocal fluorescent images showing triple immunolabeling for ChAT, CTb, and FG in the rostral PPN ( $\boldsymbol{A}$; PPNr) and (DS- the striatal complex. Injections in the lateral aspects of the dorsal striatum a similar pattern of labeling. Injections in the medial shell of the NA (NA-shell) produced retrograde labeling mainly in the LDT, whereas injections in the lateral shell produced labeling mainly in the rostral and caudal PPN. Scale bars: $\boldsymbol{A}, \boldsymbol{B}, 50 \mu \mathrm{m} ; \boldsymbol{C}, 500 \mu \mathrm{m}$.

thalamus). In neurons projecting to the NA, we also detected the presence of the tracer transported from the VTA (in $16.47 \%$ of neurons, corresponding to 58 double-labeled neurons of 325 retrogradely labeled neurons from the NA; Fig. $7 F$ ) and the thalamus (in $7.16 \%$ of neurons, corresponding to 21 double-labeled neurons of 293 retrogradely labeled neurons from the NA; Fig. $7 G$ ), thus indicating the existence of axon collaterals to those targets and validating the results obtained with the WGA-Cre fusion protein. Together, our results suggest that neurons in LDT are able to provide both a direct and indirect innervation (via VTA and thalamus) of the NA.

\section{Discussion}

The main findings of the present study are as follows. First, brainstem cholinergic neurons located in the PPN and LDT provide a major innervation of the dorsal striatum and NA that mainly avoids striosomes. Second, the projection is topographically organized such that the rostral PPN preferentially innervates the dorsolateral striatum, the caudal PPN equally innervates the dorsal striatum and NA, and the LDT preferentially innervates the dorsomedial striatum and NA. Third, accumbens-projecting cholinergic neurons in the LDT give rise to collaterals that innervate the dopamine midbrain and the thalamus, suggesting both a direct and indirect influence of the cholinergic brainstem on striatal regions. Finally, EM analysis revealed that cholinergic axons make synaptic contacts in the dorsal striatum and NA with spines 
Table 1. Distribution of retrogradely traced neurons in the brainstem

\begin{tabular}{|c|c|c|c|c|c|c|}
\hline & \multicolumn{3}{|c|}{ Normalized cell count (\%) } & \multicolumn{3}{|c|}{$\%$ Retrogradely traced neurons that were identified as cholinergic } \\
\hline & Rostral PPN & Caudal PPN & LDT & Rostral PPN & Caudal PPN & LDT \\
\hline DS lateral & $36.37 \pm 6.07$ & $47.32 \pm 5.8$ & $16.30 \pm 13.53$ & $76.16 \pm 6.69$ & $76.62 \pm 5.81$ & $61.07 \pm 10.37$ \\
\hline DS medial & $21.78 \pm 2.9$ & $32.9 \pm 3.65$ & $45.29 \pm 3.49$ & $60.61 \pm 8.7$ & $77.74 \pm 3.7$ & $74.67 \pm 3.4$ \\
\hline NA core & $15.3 \pm 3.44$ & $41.8 \pm 4.8$ & $42.8 \pm 5.46$ & $51.36 \pm 5.4$ & $69 \pm 2.02$ & $66.8 \pm 1.0$ \\
\hline NA shell (lateral) & $48.8 \pm 6.6$ & $46.8 \pm 5.5$ & $4.2 \pm 1.9$ & $72.25 \pm 4.3$ & $77.9 \pm 1.4$ & $59.75 \pm 20.5$ \\
\hline NA shell (medial) & $19.8 \pm 1.6$ & $45.1 \pm 6.1$ & $34.9 \pm 5.9$ & $41.15 \pm 3.4$ & $63.9 \pm 6.8$ & $73.9 \pm 8.1$ \\
\hline
\end{tabular}

Quantification of retrogradely labeled neurons in the PPN (rostral and caudal) and LDT after the injection of tracers in different regions of the striatal complex: dorsal striatum (DS) lateral $(n=3)$ and medial $(n=3)$, NA core $(n=4)$, and NA shell lateral $(n=4)$ and medial $(n=3)$. Data are expressed as the normalized cell count for the three regions sampled and the percentage of retrogradely traced neurons that were immunopositive for ChAT \pm SEM.

and dendritic shafts and that the profile of synapses and postsynaptic targets is different from that of striatal cholinergic interneurons. Our findings thus demonstrate that, in addition to cholinergic interneurons, cholinergic innervation of the striatum is derived from neurons in the PPN and LDT. The differential activity of striatal cholinergic interneurons and cholinergic neurons of the brainstem during reward-related paradigms suggest that the two cholinergic systems play different but complementary roles in the processing of information in the basal ganglia.

\section{Cholinergic transmission in the striatum}

Acetylcholine receptor activation exerts a powerful control over the striatum at both the presynaptic and postsynaptic levels. The direct stimulation of postsynaptic nicotinic and/or muscarinic receptors produces inhibition of projection neurons and excitation of certain types of interneurons (Calabresi et al., 2000; English et al., 2012). Furthermore, acetylcholine receptor activation modulates striatal glutamatergic and GABAergic transmission through the activation of presynaptic receptors on corticostriatal (Pakhotin and Bracci, 2007) and local interneuron terminals (Koós and Tepper, 2002), thus generating complex network effects on striatal circuits (Carrillo-Reid et al., 2009; Plata et al., 2013). Classically, cholinergic interneurons have been considered the only source of acetylcholine in the striatum. They produce a vast innervation over the entire striatal complex (Bolam et al., 1984; Phelps et al., 1985; Phelps and Vaughn, 1986) and provide a steady tone of acetylcholine release regulated by an intrinsic tonic level of firing (Bennett and Wilson, 1999; Bennett et al., 2000; Goldberg and Reynolds, 2011). During conditioned reward behavior, a synchronous pause in the tonic firing of putative cholinergic interneurons has been proposed to signal the probability of obtaining a behavioral outcome (Blazquez et al., 2002) and the outcome delivery (Joshua et al., 2008; for review, see Schulz and Reynolds, 2013), a mechanism that has been shown to be dependent on direct thalamic innervation (Ding et al., 2010). In contrast to the firing of cholinergic interneurons of the striatum, cholinergic neurons in the brainstem follow different dynamics: they typically show phasic increases in their relatively slow firing rate associated with brain state transitions (MenaSegovia et al., 2008), the latter being caused by increased cholinergic transmission in the thalamus (Steriade, 1996; Kezunovic et al., 2012). Furthermore, neurons in the PPN are phasically activated in response to salient stimuli (Pan and Hyland, 2005) and to cues that predict changes in the magnitude of a reward outcome (Okada et al., 2009). Thus, it is possible that cholinergic terminals in the striatum that are derived from the brainstem lead to phasic increases in acetylcholine release associated with different behavioral contexts. Therefore, our data, together with previous data, suggest a dual mode of cholinergic transmission in the striatum: the release of acetylcholine by cholinergic interneurons is tonic and interrupted by behaviorally relevant events, whereas the release of acetylcholine by cholinergic terminals from the brainstem would be phasically increased during salient events. Additional support for the dual innervation comes from the ultrastructural analysis. Our data revealed that the majority of cholinergic synapses from the brainstem are asymmetric. Previous analyses of ChAT-immunopositive synapses in the striatal complex (Wainer et al., 1984; but see Phelps et al., 1985; Phelps and Vaughn, 1986; Pickel and Chan, 1990) revealed both symmetrical and asymmetrical synapses, the later accounting for $\sim 35 \%$ in the striatum. This coincides with our data showing that two-thirds of synapses originating from cholinergic interneurons are symmetrical. Together with the fact that cholinergic synapses in other targets of the brainstem are asymmetric (Bolam et al., 1991; Bevan and Bolam, 1995; Omelchenko and Sesack, 2006), this suggests that a significant proportion of cholinergic synapses in the striatum may arise in the brainstem. Future experiments are necessary to address the impact of the direct brainstem cholinergic transmission and information processing on striatal circuits and the effect of their convergent influence through dopamine and thalamic afferents.

\section{Functional organization of the cholinergic brainstem}

Cholinergic neurons in the PPN and LDT display functional specializations that are related to their connectivity and their positions within different neuronal systems. The PPN is associated with arousal/attentional functions mainly through its projections to the intralaminar thalamic nuclei (Steriade et al., 1988; Parent and Descarries, 2008) and with motor functions through projections to structures embedded in different motor circuits (e.g., SNc, subthalamic nucleus, gigantocellular nucleus; Bolam et al., 1991; Kita and Kita, 2011; Martinez-Gonzalez et al., 2013). In contrast, the LDT is associated with limbic functions underscored by its connections with limbic structures, including the VTA (Omelchenko and Sesack, 2005), midline thalamic nuclei, and infralimbic and cingulate cortex (Cornwall et al., 1990), and its involvement in motivational behavior (Lammel et al., 2012). Furthermore, we observed recently that PPN and LDT cholinergic neurons selectively target distinct subtypes of dopamine neurons in the VTA and induce different modes of discharge in their postsynaptic targets (our unpublished data). The findings in the present study are in line with such a functional dichotomy of brainstem cholinergic neurons: PPN cholinergic neurons project preferentially to the dorsal striatum, which is involved in sensorimotor circuits and integrates inputs from the intralaminar thalamus and the SNc, whereas LDT cholinergic neurons preferentially project to the medial striatum and NA, both of which are involved in limbic functions and integrate inputs from the midline thalamus and the VTA. Our results suggest that the motor/ limbic specialization observed in dorsal/ventral striatal systems is reflected at the level of brainstem cholinergic neurons. 

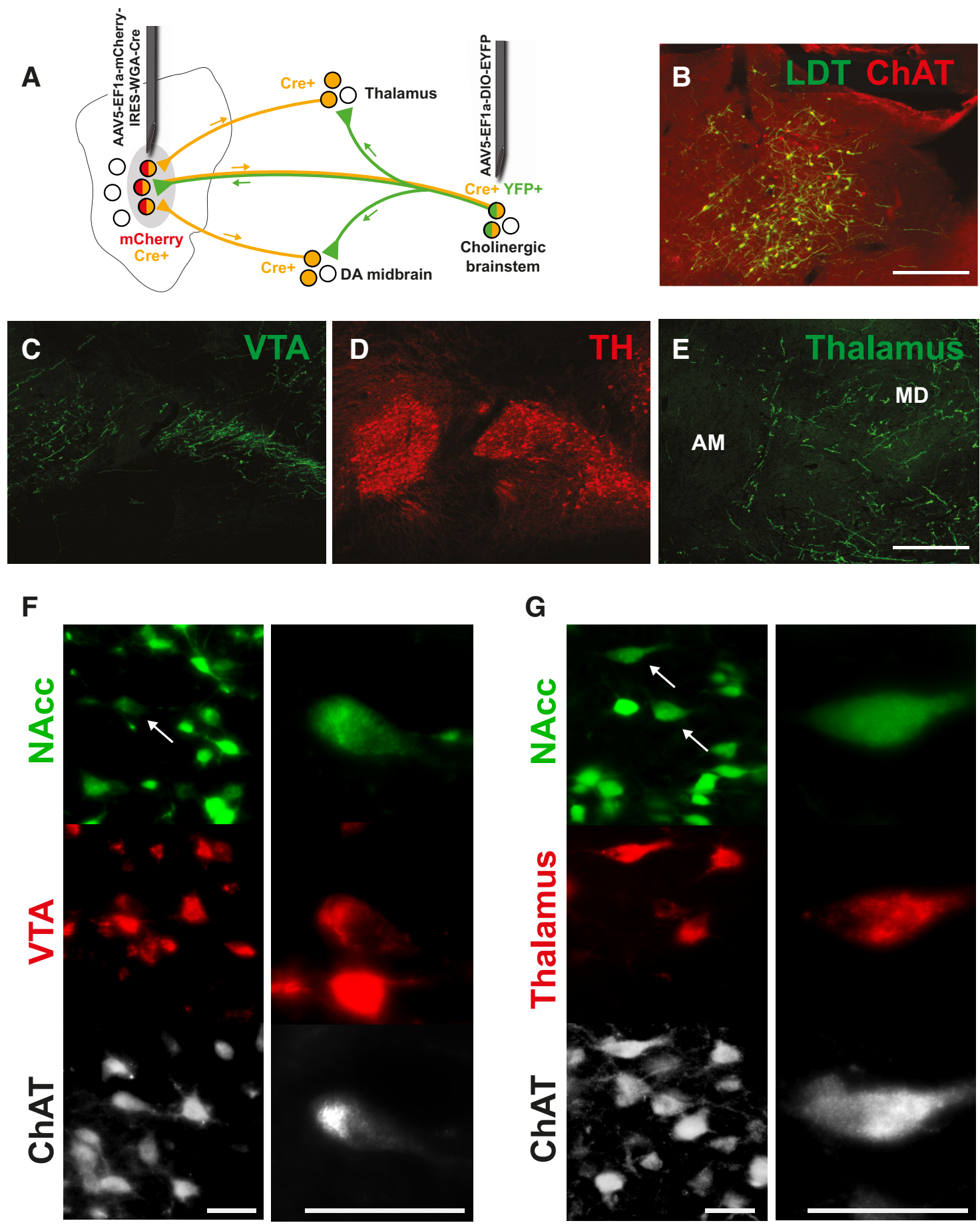

Figure 7. Labeling of axon collaterals from striatal-projecting brainstem neurons. $A$, Using a combination of two viral vectors, one of which possesses trans-neuronal retrograde capabilities, brainstem neurons that innervate striatal targets were selectively labeled, including their axon collaterals. $\boldsymbol{B}$, Neurons in the LDT that retrogradely transported WGA-Cre from the NA core expressed the YFP after the local injection of a Cre-dependent virus. $(-\boldsymbol{E}$, Axon collaterals expressing YFP were detected in the VTA $(\boldsymbol{C})$, here defined by the border of the TH staining $(\boldsymbol{D})$, and in the mediodorsal (MD) but not in the anteromedial (AM) thalamus (E), also in agreement with the study by Holmstrand and Sesack (2011).F, G, Fluorescent images showing triple immunolabeling for $C$ ATA, $(\mathrm{CTb}$, and FG in the LDT after injections in the NA (FG) and the VTA $(\boldsymbol{F} ;(\mathrm{Tb})$ or the thalamus $(\boldsymbol{G} ;(\mathrm{Cb})$. Examples of neurons with triple labeling in the left panels of $\boldsymbol{F}$ and $\boldsymbol{G}$ (arrows) are shown at higher magnification in the right panels. Scale bars: $\boldsymbol{B}, 250 \mu \mathrm{m}$; (in $\boldsymbol{E}) \boldsymbol{C}-\boldsymbol{E}, 500 \mu \mathrm{m} ; \boldsymbol{F}, \boldsymbol{G}, 50 \mu \mathrm{m}$.

\section{Noncholinergic projections from the PPN and LDT to the striatal complex}

The PPN and LDT are heterogeneous structures composed of glutamatergic and GABAergic neurons (Mena-Segovia et al., 2009; Wang and Morales, 2009), in addition to the cholinergic neurons. These noncholinergic populations have been shown to project to many of the targets of the cholinergic neurons, includ- ing the subthalamic nucleus (Bevan and Bolam, 1995; Kita and Kita, 2011; Martinez-Gonzalez et al., 2013), the substantia nigra (Futami et al., 1995; Charara et al., 1996), the thalamus (BarrosoChinea et al., 2011), and the gigantocellular nucleus (MartinezGonzalez et al., 2013), among others. We observed that a proportion of striatal-projecting PPN and LDT neurons were immunonegative for ChAT, suggesting that they may be gluta- 
matergic or GABAergic. This opens the possibility that other brainstem neurotransmitters with distinct dynamics may influence the activity of striatal microcircuits. Furthermore, although the presence of glutamate in cholinergic terminals is only found in $<5 \%$ of cholinergic neurons (Wang and Morales, 2009), additional studies should address the possibility of cotransmission from brainstem afferents and potential heterogeneous effects on postsynaptic targets.

\section{Conclusions}

In summary, we show that cholinergic neurons in the PPN and LDT have widespread, topographically organized projections to the striatal complex in which synapses are formed with both spines and dendritic shafts. Our results further suggest that the influence of the cholinergic brainstem on the striatal complex involves direct and indirect projections arising from the same set of neurons, the latter mediated by dopamine and thalamic neurons. The convergent activity on striatal microcircuits is likely to have varied and complex effects on striatal information processing. Evidence of a second major source of acetylcholine in the striatum suggests that the functional studies need to be interpreted in a broader context.

\section{References}

Aosaki T, Tsubokawa H, Ishida A, Watanabe K, Graybiel AM, Kimura M (1994) Responses of tonically active neurons in the primate's striatum undergo systematic changes during behavioral sensorimotor conditioning. J Neurosci 14:3969-3984. Medline

Apicella P, Legallet E, Trouche E (1997) Responses of tonically discharging neurons in the monkey striatum to primary rewards delivered during different behavioral states. Exp Brain Res 116:456-466. CrossRef Medline

Barroso-Chinea P, Rico AJ, Conte-Perales L, Gómez-Bautista V, Luquin N, Sierra S, Roda E, Lanciego JL (2011) Glutamatergic and cholinergic pedunculopontine neurons innervate the thalamic parafascicular nucleus in rats: changes following experimental parkinsonism. Brain Struct Funct 216:319-330. CrossRef Medline

Bennett BD, Wilson CJ (1999) Spontaneous activity of neostriatal cholinergic interneurons in vitro. J Neurosci 19:5586-5596. Medline

Bennett BD, Callaway JC, Wilson CJ (2000) Intrinsic membrane properties underlying spontaneous tonic firing in neostriatal cholinergic interneurons. J Neurosci 20:8493-8503. Medline

Bevan MD, Bolam JP (1995) Cholinergic, GABAergic, and glutamateenriched inputs from the mesopontine tegmentum to the subthalamic nucleus in the rat. J Neurosci 15:7105-7120. Medline

Blaha CD, Winn P (1993) Modulation of dopamine efflux in the striatum following cholinergic stimulation of the substantia nigra in intact and pedunculopontine tegmental nucleus-lesioned rats. J Neurosci 13:10351044. Medline

Blaha CD, Allen LF, Das S, Inglis WL, Latimer MP, Vincent SR, Winn P (1996) Modulation of dopamine efflux in the nucleus accumbens after cholinergic stimulation of the ventral tegmental area in intact, pedunculopontine tegmental nucleus-lesioned, and laterodorsal tegmental nucleus-lesioned rats. J Neurosci 16:714-722. Medline

Blazquez PM, Fujii N, Kojima J, Graybiel AM (2002) A network representation of response probability in the striatum. Neuron 33:973-982. CrossRef Medline

Bolam JP, Wainer BH, Smith AD (1984) Characterization of cholinergic neurons in the rat neostriatum. A combination of choline acetyltransferase immunocytochemistry, Golgi-impregnation and electron microscopy. Neuroscience 12:711-718. CrossRef Medline

Bolam JP, Francis CM, Henderson Z (1991) Cholinergic input to dopaminergic neurons in the substantia nigra: a double immunocytochemical study. Neuroscience 41:483-494. CrossRef Medline

Cachope R, Mateo Y, Mathur BN, Irving J, Wang HL, Morales M, Lovinger DM, Cheer JF (2012) Selective activation of cholinergic interneurons enhances accumbal phasic dopamine release: setting the tone for reward processing. Cell Rep 2:33-41. CrossRef Medline

Calabresi P, Centonze D, Gubellini P, Pisani A, Bernardi G (2000)
Acetylcholine-mediated modulation of striatal function. Trends Neurosci 23:120-126. CrossRef Medline

Carrillo-Reid L, Tecuapetla F, Ibáñez-Sandoval O, Hernández-Cruz A, Galarraga E, Bargas J (2009) Activation of the cholinergic system endows compositional properties to striatal cell assemblies. J Neurophysiol 101: 737-749. CrossRef Medline

Charara A, Smith Y, Parent A (1996) Glutamatergic inputs from the pedunculopontine nucleus to midbrain dopaminergic neurons in primates: Phaseolus vulgaris-leucoagglutinin anterograde labeling combined with postembedding glutamate and GABA immunohistochemistry. J Comp Neurol 364:254-266. CrossRef Medline

Cornwall J, Cooper JD, Phillipson OT (1990) Afferent and efferent connections of the laterodorsal tegmental nucleus in the rat. Brain Res Bull 25:271-284. CrossRef Medline

Ding JB, Guzman JN, Peterson JD, Goldberg JA, Surmeier DJ (2010) Thalamic gating of corticostriatal signaling by cholinergic interneurons. Neuron 67:294-307. CrossRef Medline

English DF, Ibanez-Sandoval O, Stark E, Tecuapetla F, Buzsáki G, Deisseroth K, Tepper JM, Koos T (2012) GABAergic circuits mediate the reinforcement-related signals of striatal cholinergic interneurons. Nat Neurosci 15:123-130. CrossRef Medline

Erro E, Lanciego JL, Giménez-Amaya JM (1999) Relationships between thalamostriatal neurons and pedunculopontine projections to the thalamus: a neuroanatomical tract-tracing study in the rat. Exp Brain Res 127:162-170. CrossRef Medline

Futami T, Takakusaki K, Kitai ST (1995) Glutamatergic and cholinergic inputs from the pedunculopontine tegmental nucleus to dopamine neurons in the substantia nigra pars compacta. Neurosci Res 21:331-342. CrossRef Medline

Galarraga E, Hernández-López S, Reyes A, Miranda I, Bermudez-Rattoni F, Vilchis C, Bargas J (1999) Cholinergic modulation of neostriatal output: a functional antagonism between different types of muscarinic receptors. J Neurosci 19:3629-3638. Medline

Goldberg JA, Reynolds JN (2011) Spontaneous firing and evoked pauses in the tonically active cholinergic interneurons of the striatum. Neuroscience 198:27-43. CrossRef Medline

Goldberg JA, Ding JB, Surmeier DJ (2012) Muscarinic modulation of striatal function and circuitry. Handb Exp Pharmacol 208:223-241. CrossRef Medline

Gould E, Woolf NJ, Butcher LL (1989) Cholinergic projections to the substantia nigra from the pedunculopontine and laterodorsal tegmental nuclei. Neuroscience 28:611-623. CrossRef Medline

Gradinaru V, Zhang F, Ramakrishnan C, Mattis J, Prakash R, Diester I, Goshen I, Thompson KR, Deisseroth K (2010) Molecular and cellular approaches for diversifying and extending optogenetics. Cell 141:154-165. CrossRef Medline

Graveland GA, DiFiglia M (1985) The frequency and distribution of medium-sized neurons with indented nuclei in the primate and rodent neostriatum. Brain Res 327:307-311. CrossRef Medline

Graybiel AM, Ragsdale CW Jr (1978) Histochemically distinct compartments in the striatum of human, monkeys, and cat demonstrated by acetylthiocholinesterase staining. Proc Natl Acad Sci U S A 75:57235726. CrossRef Medline

Hallanger AE, Wainer BH (1988) Ascending projections from the pedunculopontine tegmental nucleus and the adjacent mesopontine tegmentum in the rat. J Comp Neurol 274:483-515. CrossRef Medline

Hernández-López S, Góngora-Alfaro JL, Martínez-Fong D, Aceves J (1992) A cholinergic input to the substantia nigra pars compacta increases striatal dopamine metabolism measured by in vivo voltammetry. Brain Res 598:114-120. CrossRef Medline

Holmstrand EC, Sesack SR (2011) Projections from the rat pedunculopontine and laterodorsal tegmental nuclei to the anterior thalamus and ventral tegmental area arise from largely separate populations of neurons. Brain Struct Funct 216:331-345. CrossRef Medline

Joshua M, Adler A, Mitelman R, Vaadia E, Bergman H (2008) Midbrain dopaminergic neurons and striatal cholinergic interneurons encode the difference between reward and aversive events at different epochs of probabilistic classical conditioning trials. J Neurosci 28:1167311684. CrossRef Medline

Kawaguchi Y, Wilson CJ, Augood SJ, Emson PC (1995) Striatal interneurones: chemical, physiological and morphological characterization. Trends Neurosci 18:527-535. CrossRef Medline 
Kezunovic N, Hyde J, Simon C, Urbano FJ, Williams DK, Garcia-Rill E (2012) Gamma band activity in the developing parafascicular nucleus. J Neurophysiol 107:772-784. CrossRef Medline

Kimura M, Rajkowski J, Evarts E (1984) Tonically discharging putamen neurons exhibit set-dependent responses. Proc Natl Acad Sci U S A 81: 4998-5001. CrossRef Medline

Kita T, Kita H (2011) Cholinergic and non-cholinergic mesopontine tegmental neurons projecting to the subthalamic nucleus in the rat. Eur J Neurosci 33:433-443. CrossRef Medline

Kobayashi S, Nakamura Y (2003) Synaptic organization of the rat parafascicular nucleus, with special reference to its afferents from the superior colliculus and the pedunculopontine tegmental nucleus. Brain Res 980: 80-91. CrossRef Medline

Koós T, Tepper JM (2002) Dual cholinergic control of fast-spiking interneurons in the neostriatum. J Neurosci 22:529-535. Medline

Lacey MG, Calabresi P, North RA (1990) Muscarine depolarizes rat substantia nigra zona compacta and ventral tegmental neurons in vitro through M1-like receptors. J Pharmacol Exp Ther 253:395-400. Medline

Lammel S, Lim BK, Ran C, Huang KW, Betley MJ, Tye KM, Deisseroth K, Malenka RC (2012) Input-specific control of reward and aversion in the ventral tegmental area. Nature 491:212-217. CrossRef Medline

Martinez-Gonzalez C, Bolam JP, Mena-Segovia J (2011) Topographical organization of the pedunculopontine nucleus. Front Neuroanat 5:22. Medline

Martinez-Gonzalez C, van Andel J, Bolam JP, Mena-Segovia J (2013) Divergent motor projections from the pedunculopontine nucleus are differentially regulated in Parkinsonism. Brain Struct Funct. Advance online publication. Retrieved February 21, 2014. doi:10.1007/s00429-0130579-6. CrossRef Medline

Mena-Segovia J, Bolam JP, Magill PJ (2004) Pedunculopontine nucleus and basal ganglia: distant relatives or part of the same family? Trends Neurosci 27:585-588. CrossRef Medline

Mena-Segovia J, Sims HM, Magill PJ, Bolam JP (2008) Cholinergic brainstem neurons modulate cortical gamma activity during slow oscillations. J Physiol 586:2947-2960. CrossRef Medline

Mena-Segovia J, Micklem BR, Nair-Roberts RG, Ungless MA, Bolam JP (2009) GABAergic neuron distribution in the pedunculopontine nucleus defines functional subterritories. J Comp Neurol 515:397-408. CrossRef Medline

Morris G, Arkadir D, Nevet A, Vaadia E, Bergman H (2004) Coincident but distinct messages of midbrain dopamine and striatal tonically active neurons. Neuron 43:133-143. CrossRef Medline

Nakano K, Hasegawa Y, Tokushige A, Nakagawa S, Kayahara T, Mizuno N (1990) Topographical projections from the thalamus, subthalamic nucleus and pedunculopontine tegmental nucleus to the striatum in the Japanese monkey, Macaca fuscata. Brain Res 537:54-68. CrossRef Medline

Oakman SA, Faris PL, Kerr PE, Cozzari C, Hartman BK (1995) Distribution of pontomesencephalic cholinergic neurons projecting to substantia nigra differs significantly from those projecting to ventral tegmental area. J Neurosci 15:5859-5869. Medline

Okada K, Toyama K, Inoue Y, Isa T, Kobayashi Y (2009) Different pedunculopontine tegmental neurons signal predicted and actual task rewards. J Neurosci 29:4858-4870. CrossRef Medline

Omelchenko N, Sesack SR (2005) Laterodorsal tegmental projections to identified cell populations in the rat ventral tegmental area. J Comp Neurol 483:217-235. CrossRef Medline

Omelchenko N, Sesack SR (2006) Cholinergic axons in the rat ventral tegmental area synapse preferentially onto mesoaccumbens dopamine neurons. J Comp Neurol 494:863-875. CrossRef Medline

Pakhotin P, Bracci E (2007) Cholinergic interneurons control the excitatory input to the striatum. J Neurosci 27:391-400. CrossRef Medline

Pan WX, Hyland BI (2005) Pedunculopontine tegmental nucleus controls conditioned responses of midbrain dopamine neurons in behaving rats. J Neurosci 25:4725-4732. CrossRef Medline

Parent M, Descarries L (2008) Acetylcholine innervation of the adult rat thalamus: distribution and ultrastructural features in dorsolateral geniculate, parafascicular, and reticular thalamic nuclei. J Comp Neurol 511: 678-691. CrossRef Medline
Paxinos G, Watson C (1986) The rat brain in stereotaxic coordinates, Ed 2. San Diego: Academic.

Phelps PE, Vaughn JE (1986) Immunocytochemical localization of choline acetyltransferase in rat ventral striatum: a light and electron microscopic study. J Neurocytol 15:595-617. CrossRef Medline

Phelps PE, Houser CR, Vaughn JE (1985) Immunocytochemical localization of choline acetyltransferase within the rat neostriatum: a correlated light and electron microscopic study of cholinergic neurons and synapses. J Comp Neurol 238:286-307. CrossRef Medline

Pickel VM, Chan J (1990) Spiny neurons lacking choline acetyltransferase immunoreactivity are major targets of cholinergic and catecholaminergic terminals in rat striatum. J Neurosci Res 25:263-280. CrossRef Medline

Plata V, Duhne M, Pérez-Ortega J, Hernández-Martinez R, Rueda-Orozco P, Galarraga E, Drucker-Colín R, Bargas J (2013) Global actions of nicotine on the striatal microcircuit. Front Syst Neurosci 7:78. CrossRef Medline

Rice ME, Cragg SJ (2004) Nicotine amplifies reward-related dopamine signals in striatum. Nat Neurosci 7:583-584. CrossRef Medline

Saper CB, Loewy AD (1982) Projections of the pedunculopontine tegmental nucleus in the rat: evidence for additional extrapyramidal circuitry. Brain Res 252:367-372. CrossRef Medline

Schulz JM, Reynolds JN (2013) Pause and rebound: sensory control of cholinergic signaling in the striatum. Trends Neurosci 36:41-50. CrossRef Medline

Smith Y, Parent A (1986) Differential connections of caudate nucleus and putamen in the squirrel monkey (Saimiri sciureus) Neuroscience 18:347371. Medline

Smith Y, Paré D, Deschênes M, Parent A, Steriade M (1988) Cholinergic and non-cholinergic projections from the upper brainstem core to the visual thalamus in the cat. Exp Brain Res 70:166-180. Medline

Steriade M (1996) Arousal: revisiting the reticular activating system. Science 272:225-226. CrossRef Medline

Steriade M, Paré D, Parent A, Smith Y (1988) Projections of cholinergic and non-cholinergic neurons of the brainstem core to relay and associational thalamic nuclei in the cat and macaque monkey. Neuroscience 25:47-67. CrossRef Medline

Tepper JM, Bolam JP (2004) Functional diversity and specificity of neostriatal interneurons. Curr Opin Neurobiol 14:685-692. CrossRef Medline

Threlfell S, Lalic T, Platt NJ, Jennings KA, Deisseroth K, Cragg SJ (2012) Striatal dopamine release is triggered by synchronized activity in cholinergic interneurons. Neuron 75:58-64. CrossRef Medline

Wainer BH, Bolam JP, Freund TF, Henderson Z, Totterdell S, Smith AD (1984) Cholinergic synapses in the rat brain: a correlated light and electron microscopic immunohistochemical study employing a monoclonal antibody against choline acetyltransferase. Brain Res 308:69-76. CrossRef Medline

Wang HL, Morales M (2009) Pedunculopontine and laterodorsal tegmental nuclei contain distinct populations of cholinergic, glutamatergic and GABAergic neurons in the rat. Eur J Neurosci 29:340-358. CrossRef Medline

Wang Z, Kai L, Day M, Ronesi J, Yin HH, Ding J, Tkatch T, Lovinger DM, Surmeier DJ (2006) Dopaminergic control of corticostriatal long-term synaptic depression in medium spiny neurons is mediated by cholinergic interneurons. Neuron 50:443-452. CrossRef Medline

Witten IB, Lin SC, Brodsky M, Prakash R, Diester I, Anikeeva P, Gradinaru V, Ramakrishnan C, Deisseroth K (2010) Cholinergic interneurons control local circuit activity and cocaine conditioning. Science 330:16771681. CrossRef Medline

Witten IB, Steinberg EE, Lee SY, Davidson TJ, Zalocusky KA, Brodsky M, Yizhar O, Cho SL, Gong S, Ramakrishnan C, Stuber GD, Tye KM, Janak PH, Deisseroth K (2011) Recombinase-driver rat lines: tools, techniques, and optogenetic application to dopamine-mediated reinforcement. Neuron 72:721-733. CrossRef Medline

Xu W, Südhof TC (2013) A neural circuit for memory specificity and generalization. Science 339:1290-1295. CrossRef Medline

Zhang L, Liu Y, Chen X (2005) Carbachol induces burst firing of dopamine cells in the ventral tegmental area by promoting calcium entry through L-type channels in the rat. J Physiol 568:469-481. CrossRef Medline 